\title{
FGF4 and HGF promote differentiation of mouse bone marrow mesenchymal stem cells into hepatocytes via the MAPK pathway
}

\author{
T. Lu ${ }^{1 *}$, C. Yang ${ }^{2 *}$, H. Sun ${ }^{1}$, J. Lv ${ }^{1}$, F. Zhang ${ }^{1,3}$ and X.J. Dong ${ }^{1}$ \\ ${ }^{1}$ Clinical Laboratory Center of Shaoxing People's Hospital, \\ Shaoxing Hospital of Zhejiang University, Shaoxing, China \\ ${ }^{2}$ Affiliated Hospital of Xi' an Medical College, Xi' an, Shanxi Province, China \\ ${ }^{3}$ School of Laboratory Medicine and Life Science, Wenzhou Medical College, \\ Wenzhou, Zhejiang Province, China \\ *These authors contributed equally to this study. \\ Corresponding author: X.J. Dong \\ E-mail: dxj9666@163.com
}

Genet. Mol. Res. 13 (1): 415-424 (2014)

Received August 10, 2013

Accepted October 18, 2013

Published January 21, 2014

DOI http://dx.doi.org/10.4238/2014.January.21.9

\begin{abstract}
Our research demonstrated the potential for mouse bone marrow mesenchymal stem cells (mBMMSCs) to differentiate into hepatocytes in vitro and in vivo. However, the exact mechanism of this process remains unknown. In this study, we investigated the role of the mitogen-activated protein kinase (MAPK) cell-signaling pathway in the differentiation of mBMMSCs into hepatocytes. mBMMSCs were isolated from femurs and tibias, and hepatic differentiation was induced in Isove's modified Eagle's medium supplemented with $10 \%$ fetal bovine serum, containing human growth factor and fibroblast growth factor 4. After seven days of induction, morphological characteristics were examined. For inhibition of signaling molecular activities, the inhibitors p38 (SB203580), ERK1/2 (U0126), and MSK1 (H89) were added to the differentiation medium. Real-time polymerase chain reaction and Western blot analysis were used to evaluate the gene expression profiles
\end{abstract}


and protein expression of several markers, including the early specific markers of hepatocytes (AFP and FOXa2), phosphorylated-p38 (pp38), phosphorylated-ERK1/2 (p-ERK1/2), and phosphorylatedMSK1 (p-MSK1). Expressions of p-p38, p-ERK1/2, and p-MSK1 were effectively inhibited by their respective inhibitors. Expressions of early specific markers, $A F P$ and FOXa2, in the p38, ERK1/2, and MSK1 inhibitor-treated groups were significantly decreased compared to those of the cytokine-induced control. Notably, the expressions of $A F P$ and $F O X a 2$ in the $\mathrm{p} 38$ inhibitor group were more obviously reduced than those in the ERK1/2 inhibitor group. The MAPK signaling pathway, especially $\mathrm{p} 38$, is sufficient to drive differentiation of mBMMSCs into hepatocytes. This could increase the efficiency of hepatocyte differentiation, which would benefit clinical applications.

Key words: BMMSCs; MAPK; Hepatocyte; Hepatic differentiation; Cytokines

\section{INTRODUCTION}

Recent studies have demonstrated that bone marrow mesenchymal stem cells (BMMSCs) can be induced to generate not only the progenies of mesodermal lineages, such as adipocytes, chondrocytes, and myogenic cells, but also cells from different germ layers, such as neuronal cells and hepatocytes (Prockop, 1997; Pittenger et al., 1999; Jiang et al., 2002; Chen et al., 2006). Our laboratory previously demonstrated that mouse BMMSCs (mBMMSCs) have potential to differentiate into hepatocytes with relatively high differentiating efficiency (Chen et al., 2009). These capacities of BMMSCs have attracted tremendous attention because of their potential as a cell source for cell therapy of hepatic diseases; however, the molecular mechanisms underlying the differentiation of BMMSCs are not clear.

Mitogen-activated protein kinases (MAPKs) have been reported as the most important signaling pathway protein kinases. MAPK family transduction involves a multistep kinase cascade involving MAPK kinase kinase (MAPKKK), MAPK kinase (MAPKK), and MAPK, along with extracellular signal-regulated protein kinase (ERK), p38 kinase, and c-Jun N-terminal kinase (JNK) (Davis, 2000; Chun, 2004). Mitogen- and stress-activated kinase 1 (MSK1) is the downstream target of MAPKKs (Commodaro et al., 2010). Although p38 and ERK have been shown to play important roles in mediating stem cell proliferation and differentiation (Peng et al., 2009; Zhang et al., 2010; Li et al., 2010), their specific involvement in the differentiation into hepatocytes remains unclear. BMMSCs can be induced by fibroblast growth factor (FGF) to express not only the early specific markers of hepatocytes [ $\alpha$-fetoprotein $(A F P)$ and hepatocyte nuclear factor 3- $\beta$ (HNF-3B or FOXa2], but also the FGF receptor family FGF1IIIc and FGF2IIIc. However, whether p38 and ERK are involved in FGF-induced hepatocyte differentiation of BMMSCs needs to be clarified.

In the present study, we investigated expression levels of the early specific markers of hepatocytes, $A F P$ and $F O X a 2$, seven days after differentiation induction by FGF and human growth factor (HGF). The p38 inhibitor SB203580 and the ERK1/2 inhibitor U0126 were shown to suppress the differentiation process, and the effect was strongest in the former. 


\section{MATERIAL AND METHODS}

\section{Animals}

Four- to eight-week-old male imprinting control region (ICR) mice, obtained from the Laboratory Animal Unit of the Zhejiang Academy of Medical Sciences (Hangzhou, People's Republic of China), were used in the experiments. The animals were housed under specific pathogen-free conditions. All experimental procedures were performed according to institutional guidelines.

\section{Isolation and culture of mBMMSCs}

mBMMSCs were harvested as described previously (Chen et al., 2007). Briefly, cells were obtained from bone marrows of femurs and tibias of 8-week-old ICR mice and cultured in Isove's modified Eagle's medium (IMEM; Invitrogen Gibco, USA) supplemented with 10\% FBS (Tianhang Biological Technology, China) and 1\% penicillin/streptomycin (Invitrogen) at $37^{\circ} \mathrm{C}$ and $5 \% \mathrm{CO}_{2}$. Non-adherent cells were removed from plates $72 \mathrm{~h}$ later by changing the medium, and once the cells nearly reached confluence, they were replated at $100 \mathrm{cells} / \mathrm{cm}^{2}$.

\section{In vitro hepatic differentiation and MAPK inhibition}

BMMSCs of passage 3 were inoculated at $5 \times 10^{4}$ cells $/ \mathrm{cm}^{2}$ on six-well culture plates and divided into five groups. Briefly, in group A, the negative control group, BMMSCs were cultured in basal medium only supplemented with $10 \%$ FBS (Tianhang) and $1 \%$ penicillin/ streptomycin (Invitrogen). In group B, cells were treated with cytokines, as described previously (Pan et al., 2008), and hepatic differentiation was induced in IMEM supplemented with 10\% FBS, containing $20 \mathrm{ng} / \mathrm{mL}$ HGF (Peprotech, Rocky Hill, NJ, USA) and $10 \mathrm{ng} / \mathrm{mL}$ FGF4 (Peprotech). In the other groups, the specific inhibitors of ERK, p38, and MSK1 (H89, Beyotime, Haimen, JiangSu) were added to the induction cultures, respectively. The optimized concentrations were determined by a concentration gradient test, in which $5,10,15$, and $20 \mu \mathrm{M}$ SB203580, 5, 10, and $15 \mu \mathrm{M}$ U0126, and 10, 15, and $20 \mu \mathrm{M}$ H89 were tested. All groups were cultured at $37^{\circ} \mathrm{C}$ and $5 \% \mathrm{CO}_{2}$ for 7 days.

\section{RNA isolation and real-time polymerase chain reaction (RT-PCR)}

In brief, total RNA was isolated using TRIZOL (Invitrogen) according to manufacturer instructions, and was quantified by spectrophotometry. One-microgram total RNA samples were reverse-transcribed using the PrimeScript ${ }^{\mathrm{TM}} \mathrm{RT}$ reagent kit (TaKaRa, Dalian, China) according to manufacturer protocols. RT-PCR was performed using a SYBR PrimeScript ${ }^{\mathrm{TM}}$ RT-PCR Kit (TaKaRa) in a real-time thermal cycler system. Gene-specific primers for $\beta$-actin, AFP, and HNF-3B (also called FOXa2) were designed using the Premier software with the primers listed in Table 1 (Sangon Biotech, Shanghai, China). The PCR was carried out at $95^{\circ} \mathrm{C}$ for $30 \mathrm{~s}$, and then at $95^{\circ} \mathrm{C}$ for $5 \mathrm{~s}$, annealing at $55^{\circ} \mathrm{C}$ for $30 \mathrm{~s}$, and $72^{\circ} \mathrm{C}$ for $30 \mathrm{~s}$, for 40 cycles. The relative concentration of cDNA was determined by the standard dilution curve and normalized against that of $\beta$-actin. 
Table 1. Primer sequences used for PCR analysis.

\begin{tabular}{|c|c|}
\hline Primer sequences $\left(5^{\prime}-3^{\prime}\right)$ & Product size (bp) \\
\hline $\begin{array}{l}\text {-actin-F: TTCCTTCTTGGGTATGGAAT } \\
\beta \text {-actin-R: GAGCAATGATCTTGATCTTC }\end{array}$ & 200 \\
\hline $\begin{array}{l}\text { AFP-F: CACTGCTGCAACTCTTCGTA } \\
\text { AFP-R: CTTTGGACCCTCTTCTGTGA }\end{array}$ & 300 \\
\hline $\begin{array}{l}\text { HNF3 } 3 \text {-F: GACCTCTTCCCTTTCTACCG } \\
\text { HNF3ß-R: TTGAAGGCGTAATGGTGC }\end{array}$ & 551 \\
\hline
\end{tabular}

\section{Protein isolation and Western blot}

The total protein extraction was carried out by harvesting $2 \times 10^{5}$ serum-deprived cells $/ \mathrm{mL}$ in ice-cold lysis buffer (Beyotime). Cells were shaken at $4^{\circ} \mathrm{C}$ for $20 \mathrm{~min}$, and cooled on ice for $10 \mathrm{~min}$. The lysate was centrifuged at $14,000 \mathrm{rpm}$ for $15 \mathrm{~min}$ before the supernatant was subjected to protein concentration determination. Protein concentrations were measured using a bicinchoninic acid protein assay kit (Solarbio, Beijing, China), and were fractionated by $10 \%$ sodium dodecyl sulfate-polyacrylamide gel electrophoresis and transferred to a polyvinylidene fluoride membrane (BioRad, Hercules, CA, USA). The membrane was incubated with blocking solution [5\% nonfat dried milk in phosphate-buffered saline with $0.2 \%$ Tween-20 (PBST)] for $1 \mathrm{~h}$, and then incubated overnight at $4^{\circ} \mathrm{C}$ with the following primary antibodies: anti-AFP, anti-FOXa2, anti-ERK1/2, and anti-phospho-ERK1/2 (all from Santa Cruz Biotechnology, Dallas, TX, USA), anti-p38, anti-MSK1, anti-phospho-p38, and antiphospho-MSK1 (Abcam, Hong Kong, China). After three washes with PBST, membranes were incubated with horseradish peroxidase-conjugated secondary IgG (diluted 1:5000; Santa Cruz Biotechnology) for $1.5 \mathrm{~h}$. Immunoreactive bands were detected by Western blotting using the ECL system (Beyotime).

\section{Statistical analysis}

Statistical analysis was performed using the SPSS version 16.0 software. All experiments were repeated at least three times and the data of evaluation parameters are reported as means $\pm \mathrm{SD}$.

\section{RESULTS}

\section{Morphological changes of BMMSCs during differentiation into hepatocyte-like cells and MAPK inhibition}

Morphological changes of BM cells and BM-derived hepatocyte-like cells were observed under a phase-contrast microscopy over the course of culture. The isolated cells displayed typical fibroblast-like morphologies (Figure 1A). After 7 days of culturing in the differentiation medium, the morphology of BM cells changed to polygonal, and cell colonies formed that gradually increased in size and number. The cells in these colonies had round, clear nuclei, and granular cytoplasm (Figure 1F), whereas the cells in the negative control remained fibroblast-like with fusiform morphology (Figure 1B). Most of the cells in the differ- 
entiation medium that contained p38 inhibitor showed fibroblast-like or fusiform morphology, while others showed hepatocyte-like cell characteristic (Figure 1C). The ERK1/2 inhibitor also decreased the number of hepatocyte-like cells, although this effect was weaker than that of the p38 inhibitor (Figure 1D). Similar morphological characteristics were observed in cells cultured with the MSK1 inhibitor (Figure 1E).
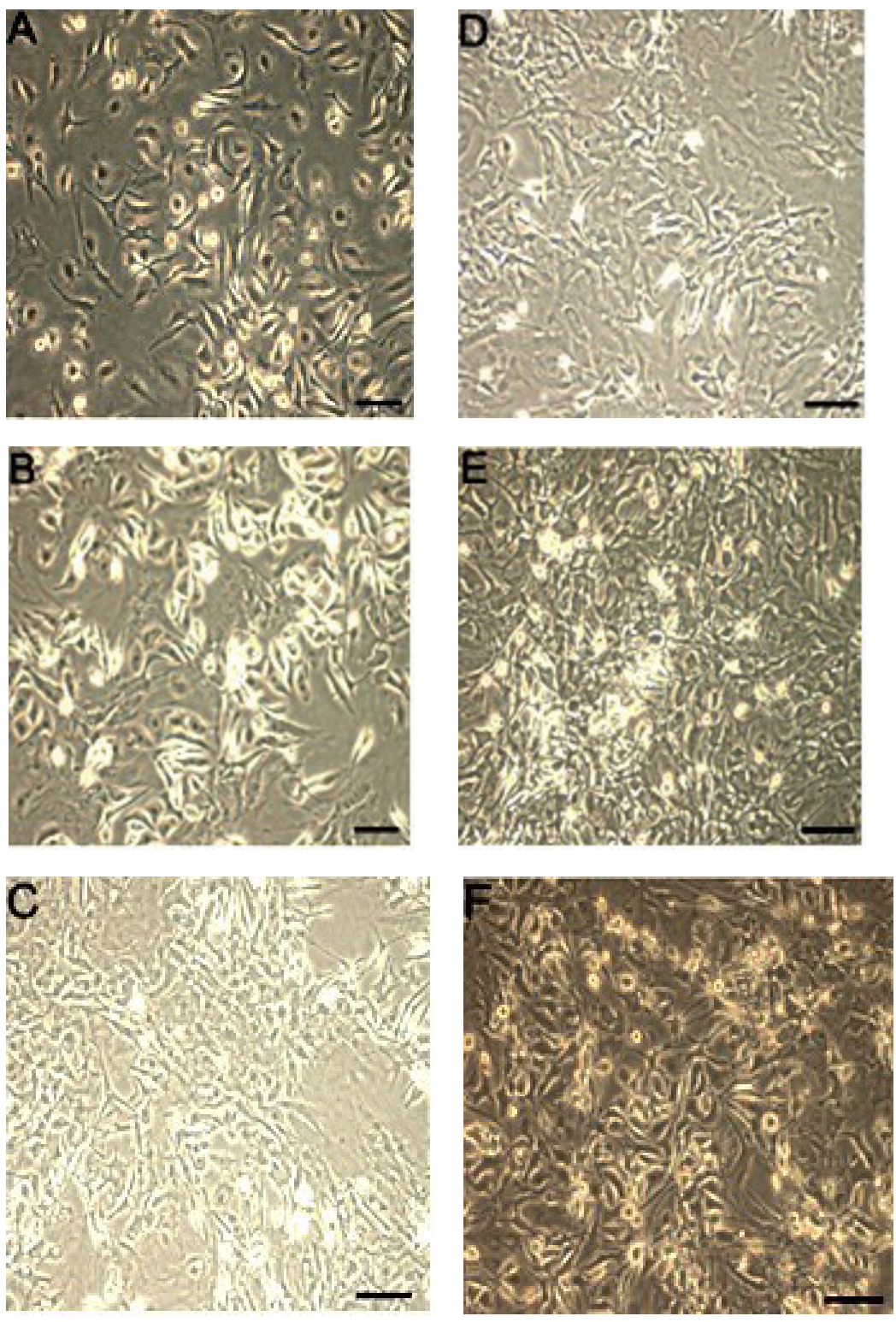

Figure 1. Morphologic changes of BMSC among groups. A. Primary culturing cells; B. negative control cells; C. P38 inhibitor group; D. ERK1/2 inhibitor group; E. MSK1 inhibitor group; F. differentiation into hepatocytelike cells. Bar: $20 \mu \mathrm{m}$. 


\section{Concentration gradient test of MAPK inhibitors}

SB203580, U0126, and H89 could all effectively decrease the phosphorylation-p38, phosphorylation-ERK1/2, and phosphorylation-MSK1, respectively, but not p38, ERK1/2, and MSK1. The efficacy of SB203580 to inhibit phosphorylation-p38 increased gradually up to $15 \mu \mathrm{M}$, although the inhibitor had similar effects at $20 \mu \mathrm{M}$. Using the same methods for the other two inhibitors, we demonstrated that the optimized concentrations of SB203580, U0126, and $\mathrm{H} 89$ were 15,10 , and $15 \mu \mathrm{M}$, respectively (Figure 2).

A

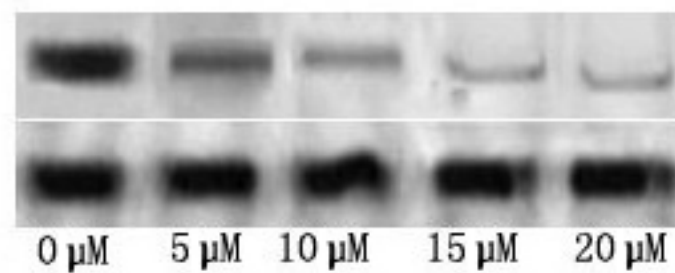

pP38

P38

B

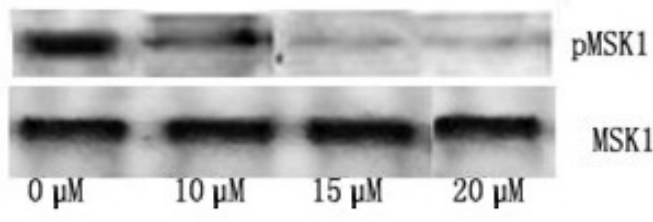

$\mathrm{C}$

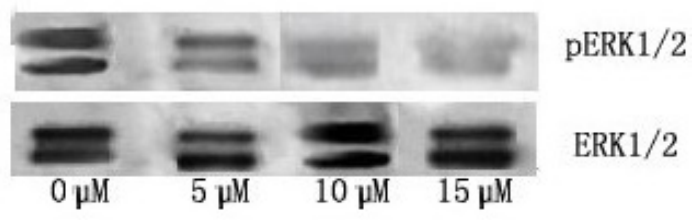

Figure 2. Concentration gradient test of MAPK inhibitor. A. $15 \mu \mathrm{M}$ P38 inhibitor had similar effects to 20 $\mu \mathrm{M}$. Finally, we have chosen SB203580 at $15 \mu \mathrm{M}$ in the followed test; B. $15 \mu \mathrm{M}$ was chosen as the optimized concentration of MSK1 inhibitor H89. C. The optimized concentration of ERK1/2 inhibitor U0126 was $10 \mu \mathrm{M}$.

\section{Effects of MAPK inhibitors on $\mathrm{AFP}$ and $\mathrm{FOX} 2$ gene expression}

The hepatic progenitor cells expressed mostly typical markers of hepatic stem cells, such as AFP, TTR, DPPIV, GGT, BG, FOXa2, CK19, and Dlk, but did not express the mature hepatocyte marker ALB (Pan et al., 2008). Therefore, the early specific markers of hepatocytes, 
$A F P$ and $F O X a 2$, were expressed 7 days after hepatic differentiation in vitro. The BMMSCs were further cultured in the induction medium supplemented with specific inhibitors of ERK (U0126), p38 MAPK (SB203580), and MSK1 (H89) at the optimized concentration indicated above. The gene expressions of $A F P$ and $F O X a 2$ were reduced to different extents, and were decreased more markedly with the p38 inhibitor than with the ERK1/2 inhibitor (Figure 3).
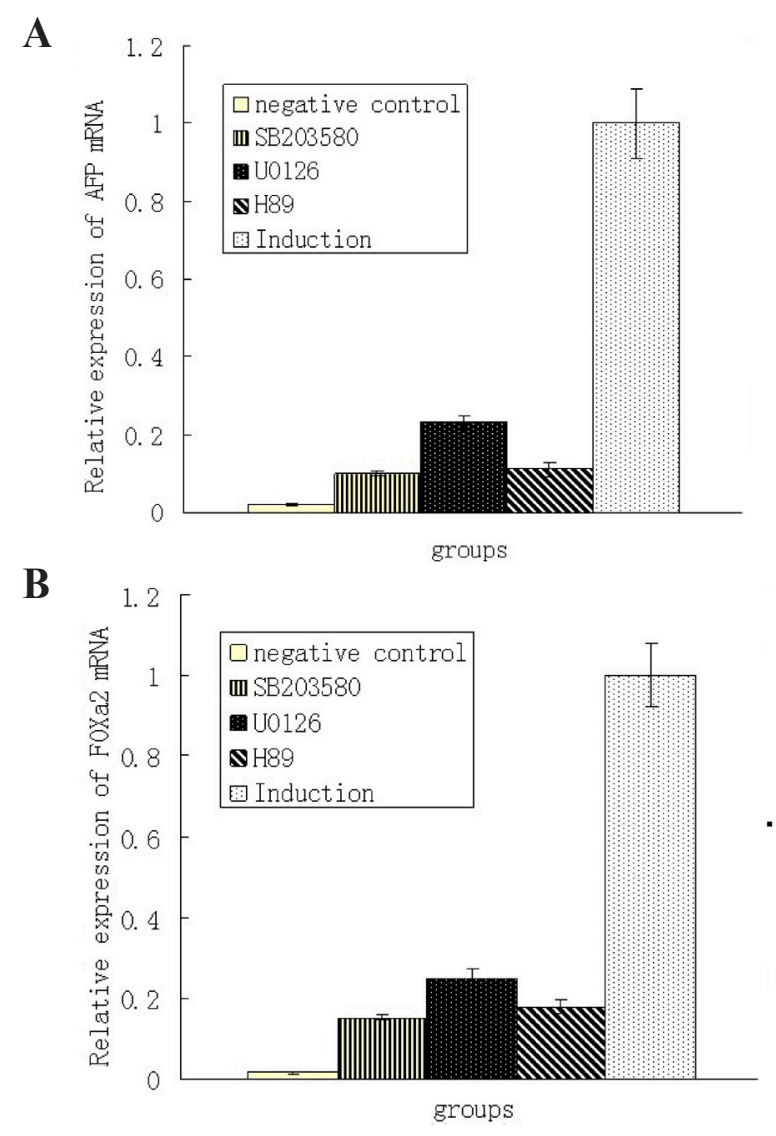

Figure 3. Effects of MAPK inhibitors on gene expression of AFP and FOXa2. A. The expression of AFP mRNA in p38, ERK1/2 and MSK1 inhibitor groups was significantly decreased than that of the cytokine differentiated induction group, and the expression of AFP mRNA in the p38 inhibitor group was decreased more obviously than that in the ERK1/2 inhibitor group. B. The expression of FOXa2 mRNA in p38, ERK1/2 and MSK1 inhibitor groups was significantly decreased than that of the cytokine differentiated induction group, and the expression of FOXa2 mRNA in the p38 inhibitor group was decreased more obviously than that in the ERK1/2 inhibitor group.

\section{Effects of MAPK inhibitors on AFP and FOXa2 protein expression}

Using the optimized concentrations of inhibitors, phosphorylation-p38, phosphorylation-ERK1/2, and phosphorylation-MSK1 were all effectively decreased. The phosphorylation of the selected signaling molecules and hepatocyte markers were measured by immunoblotting with antibodies against the corresponding molecules. Compared with the control that was cultured in basal medium only, the AFP and FOXa2 proteins were expressed in the induc- 
tion medium. When the BMMSCs were treated with the p38 inhibitor SB203580, the AFP and FOXa2 bands became weaker. The same phenomenon appeared in the other two groups when treated with ERK1/2 and MSK1 inhibitors, respectively. The bands of the p38 inhibitor group were markedly weaker than those of the ERK1/2 inhibitor group (Figure 4).

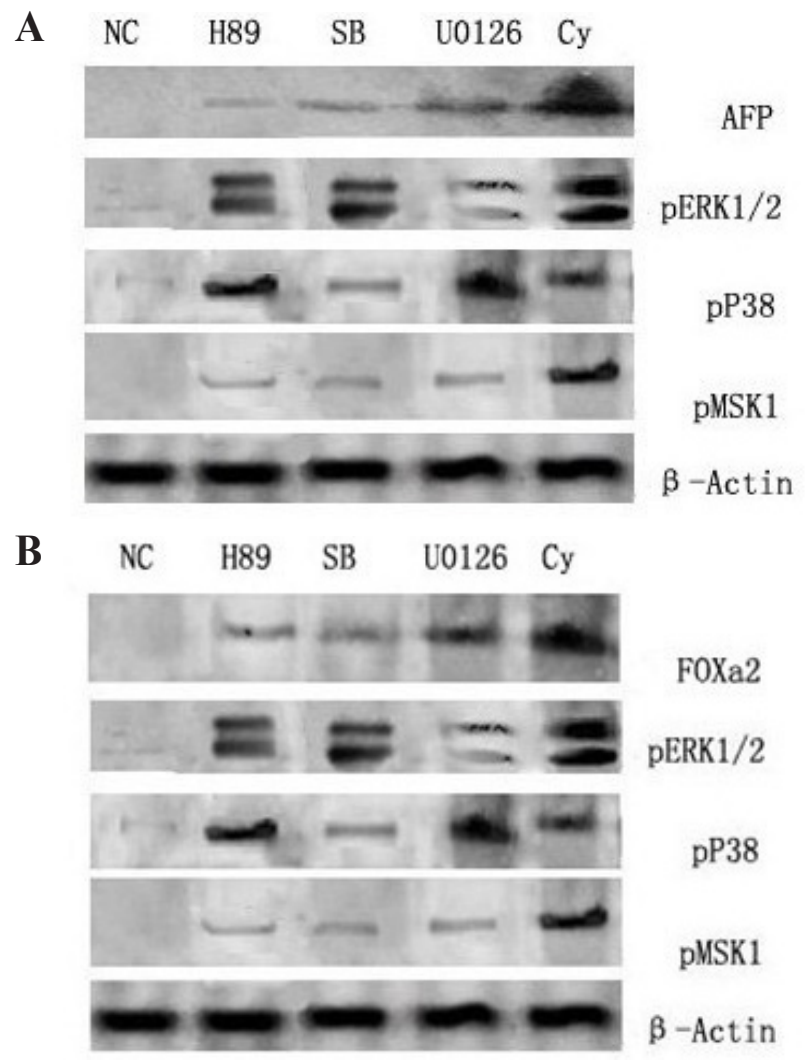

Figure 4. Effects of MAPK inhibitors on protein expression of AFP (A) and FOXa2 (B). The protein of AFP and FOXa2 was expressed at the cytokine differentiated induction group, but not in the negative control (NC) group. The levels of these two proteins were decreased by MAPK inhibitors, especially by P38 inhibitor SB203580.

MSK1 can be activated by either ERK1/2 or p38 in vivo (Darragh et al., 2010). Although both ERK1/2 and p38 are able to directly affect transcription via the phosphorylation of specific transcription factors, they are also able to induce transcription via activation of downstream kinases. In cells, MSKs are activated via a complex series of phosphorylation and auto-phosphorylation reactions occurring downstream of ERK $1 / 2$ or p $38 \alpha$. The main function of MSKs that has been identified to date is the regulation of the cAMP-response elementdependent-immediate early genes downstream of MAPK signaling (McCoy et al., 2007).

\section{DISCUSSION}

Liver transplantation is one of the most effective treatments currently used in patients 
with acute hepatic failure or end-stage liver disease. Although hepatocyte transplantation has emerged as a promising and less aggressive method to treat liver disease, it is difficult to obtain freshly isolated hepatocytes. Recent studies have demonstrated that BMMSCs can be induced to generate not only the progenies of mesodermal lineages, but also cells from different germ layers, such as neuronal cells and hepatocytes (Prockop, 1997; Pittenger et al., 1999; Jiang et al., 2002; Chen et al., 2006). Our laboratory has demonstrated that mBMMSCs have the potential to differentiate into hepatocytes with high differentiating efficiency. However, the molecular mechanisms underlying the differentiation of BMMSCs are not clear. MAPK signaling pathways have been shown to play an important role in mediating stem cell proliferation and differentiation (Peng et al., 2009; Zhang et al., 2010; Li et al., 2010). MAPK (JNK, p38, ERK) pathways constitute a large kinase network that regulates a variety of physiological processes, such as cell growth, differentiation, and apoptotic cell death, in response to different stimuli.

In our previous study (Pan et al., 2008), FGF4 and HGF were found to promote differentiation of mouse BMMSCs into hepatocytes. In the present study, morphologically, hepatocyte-like epithelioid cells appeared in group B at day seven, whereas the cells in the negative control remained fibroblast-like or of fusiform morphology. With respect to the expressions of genes and proteins, the early specific markers of hepatocytes, AFP and FOXa2, were expressed in group $B$ at day seven, but were not expressed in the negative control group. To validate whether FGF4 and HGF promote hepatocellular differentiation of mBMMSCs through p38 and ERK/MAPK signaling pathways, specific inhibitors of ERK (U0126), p38 MAPK (SB203580), and MSK1 (H89) were added to the induction culture. We observed that hepatocellular differentiation was suppressed by adding the ERK1/2 inhibitor to some degree. This result supports a previous study that showed that activation of ERK1/2 could promote basic FGF-induced neuronal differentiation of mouse bone marrow stromal cells (Yang et al., 2008). Most of the induced cells showed fibroblast-like or fusiform morphology, and the expressions of AFP and FOXa2 were also decreased in all MAPK inhibitor groups. Expression levels were reduced more obviously in the p38 inhibitor group than in the ERK $1 / 2$ inhibitor group. Therefore, we demonstrated that FGF4 and HGF promote the differentiation of mBMMSCs into hepatocytes via the MAPK pathway. Previous studies have shown that MAPK signaling pathways play an important role in mediating stem cell proliferation and differentiation into other cell types (Calmont et al., 2006; Binétruy et al., 2007; Wang et al., 2009; Tsai et al., 2010).

The MAPK cell-signaling pathway, especially $\mathrm{p} 38$, is sufficient to drive mBMMSCs to form hepatocytes. These findings could increase the efficiency of differentiating cells into hepatocytes, which will have substantial benefits in clinical applications.

\section{ACKNOWLEDGMENTS}

Research supported by the Zhejiang Natural Science Foundation (\#Y2090337) and the Key Disciplines at the Zhejiang Provincial and ShaoXing city level (\#GJSX-010-003).

\section{REFERENCES}

Binétruy B, Heasley L, Bost F, Caron L, et al. (2007). Concise review: regulation of embryonic stem cell lineage commitment by mitogen-activated protein kinases. Stem Cells 25: 1090-1095.

Calmont A, Wandzioch E, Tremblay KD, Minowada G, et al. (2006). An FGF response pathway that mediates hepatic gene induction in embryonic endoderm cells. Dev. Cell 11: 339-348. 
Chen Y, Dong XJ, Zhang GR, Shao JZ, et al. (2006). Transdifferentiation of mouse BM cells into hepatocyte-like cells. Cytotherapy 8: 381-389.

Chen Y, Dong XJ, Zhang GR, Shao JZ, et al. (2007). In vitro differentiation of mouse bone marrow stromal stem cells into hepatocytes induced by conditioned culture medium of hepatocytes. J. Cell. Biochem. 102: 52-63.

Chen Y, Pan RL, Zhang XL, Shao JZ, et al. (2009). Induction of hepatic differentiation of mouse bone marrow stromal stem cells by the histone deacetylase inhibitor VPA. J. Cell. Mol. Med. 13: 2582-2592.

Chun JS (2004). Expression, activity, and regulation of MAP kinases in cultured chondrocytes. Methods Mol. Med. 100: 291-306.

Commodaro AG, Bombardieri CR, Peron JP, Saito KC, et al. (2010). p38\{alpha\} MAP kinase controls IL-17 synthesis in Vogt-Koyanagi-Harada syndrome and experimental autoimmune uveitis. Invest. Ophthalmol. Vis. Sci. 51: 35673574.

Darragh J, Ananieva O, Courtney A, Elcombe S, et al. (2010). MSK1 regulates the transcription of IL-1ra in response to TLR activation in macrophages. Biochem. J. 425: 595-602.

Davis RJ (2000). Signal transduction by the JNK group of MAP kinases. Cell 103: 239-252.

Jiang Y, Jahagirdar BN, Reinhardt RL, Schwartz RE, et al. (2002). Pluripotency of mesenchymal stem cells derived from adult marrow. Nature 418: 41-49.

Li J, Zhao Z, Liu J, Huang N, et al. (2010). MEK/ERK and p38 MAPK regulate chondrogenesis of rat bone marrow mesenchymal stem cells through delicate interaction with TGF-beta1/Smads pathway. Cell Prolif. 43: 333-343.

McCoy CE, MacDonald A, Morrice NA, Campbell DG, et al. (2007). Identification of novel phosphorylation sites in MSK1 by precursor ion scanning MS. Biochem. J. 402: 491-501.

Pan RL, Chen Y, Xiang LX, Shao JZ, et al. (2008). Fetal liver-conditioned medium induces hepatic specification from mouse bone marrow mesenchymal stromal cells: a novel strategy for hepatic transdifferentiation. Cytotherapy 10: 668-675.

Peng S, Zhou G, Luk KD, Cheung KM, et al. (2009). Strontium promotes osteogenic differentiation of mesenchymal stem cells through the Ras/MAPK signaling pathway. Cell. Physiol. Biochem. 23: 165-174.

Pittenger MF, Mackay AM, Beck SC, Jaiswal RK, et al. (1999). Multilineage potential of adult human mesenchymal stem cells. Science 284: 143-147.

Prockop DJ (1997). Marrow stromal cells as stem cells for nonhematopoietic tissues. Science 276: 71-74.

Tsai SC, Chen CP, Su TH, Kau MM, et al. (2010). Involvement of ERK phosphorylation in the prevention of ischemiainduced ovarian follicular depletion by stem cells. Chin. J. Physiol. 53: 167-177.

Wang Y, Weil BR, Herrmann JL, Abarbanell AM, et al. (2009). MEK, p38, and PI-3K mediate cross talk between EGFR and TNFR in enhancing hepatocyte growth factor production from human mesenchymal stem cells. Am. J. Physiol. Cell Physiol. 297: C1284-C1293.

Yang H, Xia YY, Lu SQ, Soong TW, et al. (2008). basic fibroblast growth factor-induced neuronal differentiation of mouse bone marrow stromal cells requires FGFR-1, MAPK/ERK, and transcription factor AP-1. J. Biol. Chem. 283: 5287-5295.

Zhang A, Wang Y, Ye Z, Xie H, et al. (2010). Mechanism of TNF-alpha-induced migration and hepatocyte growth factor production in human mesenchymal stem cells. J. Cell. Biochem. 111: 469-475. 